\title{
Insatisfação corporal em adolescentes: um estudo de base populacional
}

\section{Body dissatisfaction among adolescents: a population-based study}

Samuel de Carvalho Dumith ${ }^{1}$

Ana Maria Baptista Menezes ${ }^{1}$

Renata Moraes Bielemann ${ }^{1}$

Sandra Petresco ${ }^{1}$

Inácio Crochemore Mohnsan da Silva ${ }^{1}$

Rogério da Silva Linhares ${ }^{1}$

Tales Costa Amorim ${ }^{1}$

Daniel Vanti Duarte ${ }^{1}$

Cora Luíza Pavin Araújo ${ }^{1}$

Janaína Vieira dos Santos ${ }^{1}$

${ }^{1}$ Departamento de Medicina Social, Faculdade de

Medicina, Universidade Federal de Pelotas. Rua Marechal Deodoro 1160/3o piso, Centro. 96020-220 Pelotas RS.

scdumith@yahoo.com.br
Abstract The scope of this study was to evaluate body dissatisfaction among adolescents, and to explore differences according to sex, socioeconomic status and body mass index. 4325 individuals aged 14-15 years old from Pelotas, Brazil, were studied. Body dissatisfaction was evaluated comparing the desired image with the perceived image, according to the Tiggemann \& Wilson-Barret silhouette scale. The nutritional status was categorized in accordance with World Health Organization criteria, whereas socioeconomic status was evaluated using classifications of the Brazilian Association of Research Institutes. It was found that $27.6 \%$ of teens were overweight. Body dissatisfaction, appraised by the silhouette scale, affected $51 \%$ of boys and $65.6 \%$ of girls. The economic and nutritional status was associated with the outcome. Most overweight adolescents wished to have a slimmer silhouette than the perceived one. The economic level modified the effect of nutritional status on body dissatisfaction. Adolescents from the wealthier economic classes who were not in the ideal weight range manifested greater body dissatisfaction than the less affluent individuals and, irrespective of economic status, girls are more dissatisfied with excess body weight and boys with slimness.

Key words Body image, Nutritional status, Weight perception, Body mass index, Adolescent
Resumo O propósito deste estudo foi avaliar a insatisfação corporal em adolescentes e explorar diferenças conforme sexo, nível econômico e indice de massa corporal. Foram estudados 4.325 indivíduos de 14 a 15 anos da cidade de Pelotas, Brasil. A insatisfação corporal foi avaliada comparando a imagem desejada com a imagem percebida, de acordo com escala de silhuetas de Tiggemann e WilsonBarret. O estado nutricional foi categorizado conforme proposto pela Organização Mundial da Saúde, enquanto o nível socioeconômico foi avaliado segundo classificação da Associação Brasileira de Empresas de Pesquisas. Verificou-se que 27,6\% dos adolescentes estavam acima do peso. A insatisfação corporal, avaliada pela escala de silhuetas, atingiu 51,0\% dos meninos e $65,6 \%$ das meninas. O nível econômico e o estado nutricional estiveram associados com o desfecho. A maioria dos adolescentes com excesso de peso desejava ter uma silhueta menor do que a percebida. O nível econômico modificou o efeito do estado nutricional sobre a insatisfação corporal. Adolescentes das classes econômicas mais altas fora do peso ideal mostraram maior insatisfação corporal do que os mais pobres $e$, independente do nível econômico, as meninas estão mais insatisfeitas com o excesso de peso e os meninos com a magreza.

Palavras-chave Imagem corporal, Percepção de peso, Índice de massa corporal, Adolescente 


\section{Introdução}

A forma como o adolescente percebe a própria imagem corporal traz conseqüências para a sua saúde física e mental, com possíveis repercussões em suas relações pessoais. A Pesquisa Nacional de Saúde do Escolar ${ }^{1}$, desenvolvida com estudantes de 13 a 15 das 26 capitais federais mais o Distrito Federal, revelou que $40 \%$ dos adolescentes entrevistados se apercebem como muito magro/ magro ou gordo/muito gordo, sendo que proporção maior dos que se acham gordo/muito gordo foi encontrada para adolescentes do sexo feminino.

A insatisfação corporal é representada pelo desejo do adolescente de que seu corpo seja diferente da forma como o percebe, existindo uma avaliação negativa do próprio corpo. A insatisfação corporal passa por uma valorização cultural que varia de acordo com sexo, índice de massa corporal e nível econômico.

O fato de desejar ter um corpo diferente do seu estado nutricional é uma importante informação para os profissionais de saúde, e poderia ser utilizada no planejamento de ações que potencializem a adoção de comportamentos saudáveis ao adolescente. Além disso, este tipo de medida é utilizado em inquéritos epidemiológicos, sendo tratada como marcador de risco para distúrbios alimentares e outros transtornos ${ }^{2,3}$.

Neste contexto, o objetivo do presente estudo foi avaliar a insatisfação corporal entre adolescentes pertencentes a uma coorte de nascimentos a partir de uma escala de silhuetas. Além disso, diferenças por sexo e nível socioeconômico também foram analisadas.

\section{Metodologia}

Foi realizado um estudo transversal cuja população em estudo consiste de adolescentes pertencentes à Coorte de Nascimentos de 1993 de Pelotas, RS, Brasil. Estes adolescentes são acompanhados desde o nascimento ${ }^{4}$, sendo que a última visita ocorreu em 2008, quando se tentou acompanhar todos os adolescentes pertencentes a coorte, $($ idade média $=14,7$; desvio-padrão $=0,3$ ). Pelotas é uma cidade localizada no sul do Brasil, com aproximadamente 350 mil habitantes, que apresenta um alto índice de desenvolvimento humano (IDH = 0,82), similar ao estado do Rio Grande do Sul, onde está situada. Os dados foram coletados por meio de entrevistas domiciliares, aplicadas aos adolescentes e suas mães ou responsáveis. Além disso, todos os adolescentes entrevistados eram convidados a comparecer à "Central de Medidas" para a coleta de medidas biológicas (sangue e saliva) e antropométricas (peso, altura e pregas cutâneas). O estudo foi aprovado pelo Comitê de Ética em Pesquisa da Faculdade de Medicina da Universidade Federal de Pelotas, e os indivíduos que aceitassem participar do estudo deveriam assinar um termo de consentimento livre e esclarecido.

Para constituir a equipe de trabalho, as entrevistadoras passaram por um processo de seleção, treinamento de 40 horas e avaliação baseada nos questionários e manuais a serem utilizados. Adicionalmente, algumas entrevistadoras foram recrutadas e padronizadas para compor a equipe de coleta das medidas. Durante os oito meses de trabalho de campo, foram desenvolvidas duas repadronizações para garantir a qualidade das medidas antropométricas.

Os adolescentes foram pesados com balanças eletrônicas Tanita ${ }^{\circledR}$, com precisão de $100 \mathrm{~g}$, e sua estatura medida com estadiômetro de alumínio, com precisão de $1 \mathrm{~mm}$. Por meio destas mensurações calculou-se o IMC dos adolescentes, classificando-os em quatro grupos de estado nutricional (magro, normal, sobrepeso e obeso), conforme as curvas de referência preconizadas pela Organização Mundial da Saúde (OMS) ${ }^{5}$. O nível socioeconômico foi avaliado segundo a classificação proposta pela Associação Brasileira de Empresas de Pesquisas (Abep) ${ }^{6}$, a qual divide os indivíduos em classes de A (mais ricos) a $\mathrm{E}$ (mais pobres). Essa classificação leva em consideração a escolaridade do chefe da família, a posse de bens, e a presença de empregada doméstica mensalista trabalhando para a família.

O desfecho foi estudado (insatisfação corporal) comparando-se a imagem corporal desejada com a imagem corporal percebida. A imagem corporal, percebida e desejada, foi mensurada por meio da escala de silhuetas de Tiggemann e Wilson-Barret ${ }^{7}$ (Figura 1). Esta escala contém nove figuras do sexo masculino e feminino, ordenadas da menor para a maior silhueta. Primeiramente, os adolescentes escolhiam a figura a qual acreditavam parecer; a seguir, elegiam a silhueta que desejavam ter. Se a resposta das duas questões fosse a mesma silhueta, considerava-se aquele adolescente como satisfeito com sua imagem corporal. Caso contrário, o adolescente era considerado insatisfeito com a própria imagem corporal, podendo desejar ter a silhueta maior ou menor, de acordo com a resposta à segunda pergunta. 

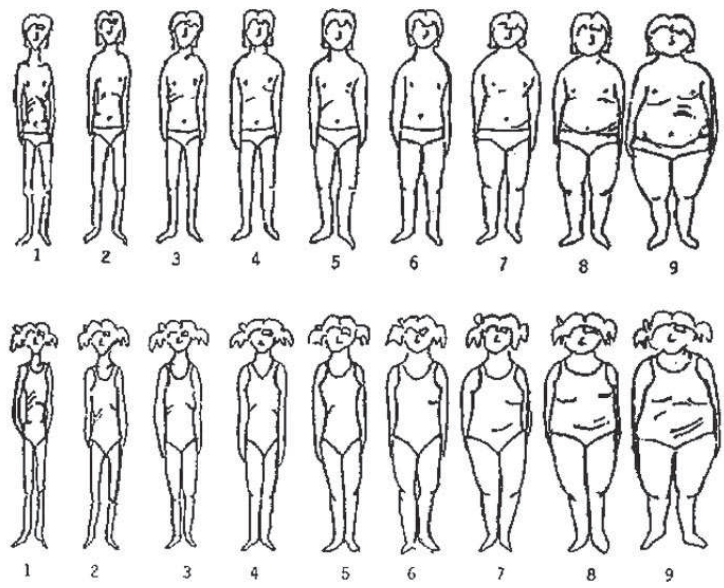

Figura 1. Escala de silhuetas de Tiggemann \& Wilson-Barret (1998). acompanhamento de $85,7 \%$ em relação aos indivíduos que fazem parte da Coorte de Nascimentos de 1993 de Pelotas, RS, Brasil. Os indivíduos estudados não diferiram da coorte original quanto às variáveis sexo e nível econômico. Pouco mais da metade $(51,2 \%)$ eram do sexo feminino. Obteve-se informação de peso e altura para 4100 adolescentes.

A Tabela 1 mostra a descrição dos adolescentes entrevistados. A maioria de ambos os sexos pertencia à classe econômica $\mathrm{C}$ e $27,6 \%$ encontravam-se acima do peso. O percentual de adolescentes com excesso de peso (sobrepeso e obesidade) foi maior no sexo masculino $(\mathrm{p}<0,001)$. A insatisfação corporal, avaliada pela escala de silhuetas, atingiu 51,0\% dos meninos e 65,6\% das meninas ( $\mathrm{p}<0,001)$, sendo que em torno de $45 \%$ das meninas desejavam ter uma silhueta menor. A correlação entre a imagem percebida e a desejada foi moderada com valor de $r=0,39$ (concordância $=41 \%$; $\mathrm{kappa}=0,21$ ).

A Tabela 2 mostra a distribuição da insatisfação corporal, de acordo com a classe econômica e o estado nutricional. Observa-se que a proporção de meninos das classes D/E que desejam uma silhueta maior foi o dobro daqueles nas classes A/B $(p<0,001)$. Entre as meninas, também foi observada maior proporção de adolescentes que gostaria de ter uma silueta maior entre aquelas pertencentes à classe econômica inferior $(\mathrm{p}<$ 0,001). Também foi observado que meninas do maior nível econômico apresentado são mais satisfeitas com a imagem corporal que as das demais categorias. Com relação ao estado nutricional, 56\% dos meninos e $42 \%$ das meninas com peso normal estavam satisfeitos com sua imagem corporal. Entre aqueles classificados como magros, cerca de três quartos gostaria de aumentar sua silhueta. Entre os classificados como obesos, mais de $90 \%$ desejavam diminuir a silhueta.

Devido à diferença de efeito do nível econômico sobre a insatisfação corporal entre os sexos (teste de interação significativo), as análises com o desfecho de forma dicotômica (insatisfeito/satisfeito) foram feitas separadamente por classes. $\mathrm{Na}$ Tabela 3, encontram-se os resultados da associação entre insatisfação corporal, medida pela escala de silhuetas, e o estado nutricional. Entre os meninos, aqueles no estágio de magreza tiveram maior probabilidade de apresentar insatisfação com a forma do corpo, comparados àqueles com peso normal, independentemente da classe econômica. Os meninos com sobrepeso pertencentes às classes $\mathrm{A} / \mathrm{B}$ e $\mathrm{C}$ também tiveram maior insatisfação que os eutróficos. Porém, o
Foram entrevistados 4325 indivíduos, o que, adicionando os óbitos, correspondeu a uma taxa de 
Tabela 1. Descrição do nível econômico, estado nutricional e insatisfação corporal de adolescentes de 14/ 15 anos conforme sexo $(\mathrm{N}=4325)$. Pelotas, Brasil, 2008.

\begin{tabular}{|c|c|c|c|c|c|}
\hline \multirow[b]{2}{*}{ Variáveis } & \multicolumn{2}{|c|}{ Masculino } & \multicolumn{2}{|c|}{ Sexo feminino } & \multirow[b]{2}{*}{$\mathbf{p}$} \\
\hline & $\mathbf{N}$ & $\%$ & $\mathbf{N}$ & $\%$ & \\
\hline Nível econômico & & & & & 0,1 \\
\hline $\mathrm{A} / \mathrm{B}$ & 573 & 27,3 & 561 & 25,5 & \\
\hline $\mathrm{C}$ & 1084 & 51,7 & 1202 & 54,7 & \\
\hline $\mathrm{D} / \mathrm{E}$ & 441 & 21,0 & 436 & 19,8 & \\
\hline Estado nutricional & & & & & $<0,001$ \\
\hline Magro & 35 & 1,8 & 26 & 1,2 & \\
\hline Normal & 1374 & 68,6 & 1534 & 73,1 & \\
\hline Sobrepeso & 389 & 19,4 & 387 & 18,5 & \\
\hline Obesidade & 204 & 10,2 & 151 & 7,2 & \\
\hline Insatisfação corporal (diferença na escala de silhuetas) & & & & & $<0,001$ \\
\hline Gostaria de ter uma silhueta menor & 576 & 27,6 & 1003 & 45,9 & \\
\hline Satisfeito com a imagem corporal & 1024 & 49,0 & 751 & 34,4 & \\
\hline Gostaria de ter uma silhueta maior & 488 & 23,4 & 430 & 19,7 & \\
\hline
\end{tabular}

Tabela 2. Associação da insatisfação corporal conforme nível econômico e estado nutricional de adolescentes de 14/ 15 anos. Pelotas, Brasil, 2008.

\begin{tabular}{|c|c|c|c|c|c|c|}
\hline \multirow[b]{2}{*}{ Variáveis } & \multicolumn{3}{|c|}{ Sexo masculino $(N=2087)$} & \multicolumn{3}{|c|}{ Sexo feminino $(\mathrm{N}=2183)$} \\
\hline & $\begin{array}{l}\text { Gostaria de ter } \\
\text { uma silhueta } \\
\text { menor }\end{array}$ & $\begin{array}{l}\text { Satisfeito com } \\
\text { a imagem } \\
\text { corporal }\end{array}$ & $\begin{array}{c}\text { Gostaria de ter } \\
\text { uma silhueta } \\
\text { maior }\end{array}$ & $\begin{array}{l}\text { Gostaria de ter } \\
\text { uma silhueta } \\
\text { menor }\end{array}$ & $\begin{array}{l}\text { Satisfeito com } \\
\text { a imagem } \\
\text { corporal }\end{array}$ & $\begin{array}{c}\text { Gostaria de ter } \\
\text { uma silhueta } \\
\text { maior }\end{array}$ \\
\hline Nível econômico & $\mathrm{p}<0,001$ & $\mathrm{p}=0,11$ & $\mathrm{p}<0,001$ & $\mathrm{p}=0,86$ & $\mathrm{p}<0,001$ & $\mathrm{p}<0,001$ \\
\hline $\mathrm{A} / \mathrm{B}$ & 32,6 & 51,7 & 15,7 & 45,4 & 42,0 & 12,6 \\
\hline $\mathrm{C}$ & 27,2 & 49,2 & 23,6 & 46,4 & 32,6 & 21,0 \\
\hline $\mathrm{D} / \mathrm{E}$ & 21,8 & 45,0 & 33,2 & 45,0 & 29,7 & 25,3 \\
\hline Estado nutricional & $\mathrm{p}<0,001$ & $\mathrm{p}<0,001$ & $\mathrm{p}<0,001$ & $\mathrm{p}<0,001$ & $\mathrm{p}<0,001$ & $\mathrm{p}<0,001$ \\
\hline Magro & 2,8 & 22,9 & 74,3 & 4,0 & 24,0 & 72,0 \\
\hline Normal & 11,8 & 56,1 & 32,1 & 32,2 & 41,8 & 26,0 \\
\hline Sobrepeso & 54,9 & 44,1 & 1,0 & 83,5 & 15,5 & 1,0 \\
\hline Obesidade & 89,2 & 10,3 & 0,5 & 94,7 & 4,7 & 0,6 \\
\hline
\end{tabular}

Tabela 3. Associação entre a insatisfação corporal e o estado nutricional de adolescentes de 14/15 anos (N=4091). Pelotas, Brasil, 2008.

\begin{tabular}{|c|c|c|c|c|c|c|}
\hline \multirow{3}{*}{$\begin{array}{c}\text { Estado } \\
\text { nutricional }\end{array}$} & \multicolumn{3}{|c|}{ Masculino } & \multicolumn{3}{|c|}{ Feminino } \\
\hline & \multicolumn{3}{|c|}{ Nível econômico } & \multicolumn{3}{|c|}{ Nível econômico } \\
\hline & $\begin{array}{c}\text { A/B } \\
\operatorname{RP}\left(\text { IC }_{95 \%}\right)\end{array}$ & $\begin{array}{c}\mathrm{C} \\
\mathrm{RP}\left(\mathrm{IC}_{95 \%}\right)\end{array}$ & $\begin{array}{c}\mathrm{D} / \mathrm{E} \\
\mathrm{RP}\left(\mathrm{IC}_{95 \%}\right)\end{array}$ & $\begin{array}{c}A / B \\
\operatorname{RP}\left(\text { IC }_{95 \%}\right)\end{array}$ & $\begin{array}{c}\mathrm{C} \\
\mathrm{RP}\left(\mathrm{IC}_{95 \%}\right)\end{array}$ & $\begin{array}{c}\mathrm{D} / \mathrm{E} \\
\mathrm{RP}\left(\mathrm{IC}_{95 \%}\right)\end{array}$ \\
\hline & $\mathrm{p}<0,001$ & $\mathrm{p}<0,001$ & $\mathrm{p}<0,001$ & $\mathrm{p}<0,001$ & $\mathrm{p}<0,001$ & $\mathrm{p}<0,001$ \\
\hline Magro & $2,01(1,12-3,59)$ & $1,65(1,26-2,16)$ & $1,74(1,35-2,24)$ & $1,21(0,59-2,49)$ & $1,19(0,85-1,66)$ & $1,56(1,44-1,70)$ \\
\hline Normal & 1,00 & 1,00 & 1,00 & 1,00 & 1,00 & 1,00 \\
\hline Sobrepeso & $1,55(1,27-1,89)$ & $1,24(1,07-1,45)$ & $1,09(0,85-1,40)$ & $1,72(1,51-1,96)$ & $1,39(1,28-1,51)$ & $1,35(1,20-1,52)$ \\
\hline Obesidade & $2,43(2,07-2,84)$ & $2,01(1,81-2,24)$ & $1,79(1,51-2,11)$ & $1,89(1,66-2,15)$ & $1,63(1,52-1,74)$ & $1,39(1,20-1,63)$ \\
\hline
\end{tabular}

RP - Razão de Prevalência; IC - Intervalo de Confiança 
mesmo não ocorreu para os meninos com sobrepeso das classes D/E. Os adolescentes obesos também estiveram mais insatisfeitos com sua imagem corporal, sendo que o grau de insatisfação foi maior para os de classe econômica superior. Entre as meninas magras, a prevalência de insatisfação foi maior apenas para as de menor status econômico. Entre aquelas acima do peso, houve maior prevalência de insatisfação do que entre as eutróficas, e esse efeito aumentou conforme o nível econômico, sendo maior para as meninas das classes $\mathrm{A} / \mathrm{B}$, assim como observado entre os meninos.

Foi conduzida análise ajustada sobre o efeito do IMC na insatisfação corporal, conforme estratos de sexo e nível econômico. Como não houve diferença nos resultados, os mesmos não foram apresentados.

\section{Discussão}

Poucos estudos avaliaram a prevalência de insatisfação corporal em adolescentes, explorando diferenças conforme sexo, nível socioeconômico e estado nutricional. Embora a literatura mostre alguns estudos sobre esta temática, a maioria destes foi realizada com tamanhos de amostras limitados e grupos específicos. São escassos os trabalhos de base populacional utilizando diferentes abordagens para investigação do tema.

Entre os pontos negativos deste estudo, além da utilização de uma escala não validada para adolescentes brasileiros, ressalta-se que a insatisfação corporal, definida a partir da diferença entre a imagem percebida e desejada, de acordo com a escala de silhuetas de Tiggemann e Wilson-Barret ${ }^{7}$, pode ser considerada um critério muito sensível, devido à amplitude desta escala (nove figuras). Pequenos desejos de mudança dos indivíduos fazem com que os mesmos sejam alocados como insatisfeitos. No entanto, análises mais conservadoras poderiam deixar de detectar a insatisfação, tratando como tal apenas situações extremas. Além disso, este tipo de definição operacional vem sendo empregada em outros estudos, com as mais diversas populações ${ }^{9-11}$.

As medidas subjetivas, como as escalas de silhuetas, além de possuírem ampla aplicabilidade em estudos populacionais, apresentam importante papel na análise de características que consideram principalmente a opinião e reflexão do próprio indivíduo, assim como na avaliação de possíveis transtornos de imagem. Neste sentido, Tabak et al. ${ }^{3}$ afirmam que as medidas subjetivas de imagem corporal estão mais associadas com o grau de satisfação com a vida dos adolescentes do que as medidas objetivas.

No presente estudo houve evidente associação entre o estado nutricional e a insatisfação corporal, para ambos os sexos. Adolescentes abaixo e acima do peso apresentaram maior grau de insatisfação corporal do que aqueles eutróficos. Esses achados são consistentes àqueles encontrados por Branco et al. ${ }^{12}$ em estudo transversal realizado em escola pública de São Paulo (SP), com jovens entre 14 e 19 anos e Corseuil et al. ${ }^{9}$, em estudo com adolescentes do sexo feminino do Rio Grande do Sul. Além disso, chama atenção que mesmo com a maior insatisfação entre aqueles com sobrepeso e obesidade, adolescentes com peso normal também apresentaram um percentual elevado deste desfecho.

Corroborando os achados do presente estudo, Wang et al. ${ }^{11}$ observaram menor insatisfação corporal entre os meninos com sobrepeso e obesos do que entre as meninas acima do peso. Conti et al. ${ }^{2}$, em estudo com adolescentes de uma escola particular de Santo André, São Paulo, verificaram associação apenas entre as meninas. Por outro lado, Haines et al. ${ }^{13}$ observaram, em estudo longitudinal, que a preocupação com o peso esteve associada ao sobrepeso nos meninos.

As meninas quando se revelam insatisfeitas, em sua maioria gostariam de ser mais magras ${ }^{10}$, enquanto que os meninos frequentemente desejam ser mais fortes, maiores, com mais múscu$\operatorname{los}^{14}$. Nossos resultados vão ao encontro da literatura com relação às meninas. No entanto, a maioria dos meninos insatisfeitos com sua imagem corporal desejava ter uma silhueta menor, diferente do suposto pela literatura. Uma possível explicação é a tendência de aumento da prevalência de excesso de peso nessa faixa etária ${ }^{15}$.

Uma variável que poderia influenciar as diferenças entre o sexo dos adolescentes é o desenvolvimento sexual, visto que meninos e meninas frequentemente atingem estágios mais avançados de maturação sexual em idades diferentes ${ }^{16}$. As tábuas de Tanner são muito utilizadas para indicar o estágio de maturação sexual ${ }^{17}$. Nesse estudo foi avaliada uma possível modificação de efeito da maturação sexual na associação entre o estado nutricional e a insatisfação corporal, porém não houve interação (dados não apresentados).

A associação entre insatisfação corporal e estado nutricional diferiu conforme os estratos de nível econômico. Embora a prevalência de insatisfação tenha sido maior entre os adolescentes pertencentes a classes econômicas inferiores, 
quando analisada de acordo com o estado nutricional, a insatisfação entre os jovens magros e aqueles acima do peso foi maior entre aqueles pertencentes a classes econômicas mais elevadas. Isso mostra que o efeito de estar abaixo ou acima do peso sobre a insatisfação corporal é bem mais evidente para adolescentes de maior nível socioeconômico. Entre os adolescentes de classe econômica inferior, o efeito do estado nutricional sobre a insatisfação corporal foi menos saliente. Estes achados podem ser atribuídos a diferenças socioculturais existentes entre as diferentes classes estudadas. Por outro lado, meninos de classes mais favorecidas podem estar mais expostos a influências midiáticas, tornando-os mais insatisfeitos com sua imagem corporal.

Todos os adolescentes de sexo masculino na categoria de magreza estiveram insatisfeitos com sua imagem corporal, independente do nível econômico. Considerando as diferenças concernentes aos níveis econômicos, verificamos maior prevalência de meninos de níveis econômicos mais baixos desejando ter uma silhueta maior e maior prevalência de adolescentes do sexo masculino de níveis mais altos desejando uma silhueta menor. Contrariando o exposto por Pereira et al. ${ }^{18}$, em estudo com escolares de nove a 15 anos, os quais observaram maior insatisfação pelo excesso de peso nos meninos das classes mais baixas e maior insatisfação pela magreza nas classes altas. Já nas meninas, o desejo por uma silhueta menor foi descrito nos diferentes níveis econômicos avaliados, assim como o observado por Pereira et al. ${ }^{18}$. Não obstante, na análise estratificada, em alguns estratos econômicos, meninas classificadas em estado nutricional de magreza não apresentaram risco de insatisfação corporal. Estes resultados reforçam que o corpo magro é mais frequentemente desejado nessa população, possivelmente por existir uma supervalorização a magreza como padrão de beleza.

Explorando a temática entre as meninas de maior nível econômico, muitas vezes considerado como um grupo de risco para insatisfação corporal, estudos com essas populações específicas apontam resultados relevantes. Dados de um estudo com estudantes do sexo feminino de um colégio particular em um município paranaense $^{12}$ evidenciaram uma prevalência de apenas 29,6\% de satisfação com o corpo, enquanto $70,4 \%$ estavam insatisfeitas. Das insatisfeitas, $66,7 \%$ gostariam de diminuir o peso e $3,7 \%$ de aumentar. Já alunas de uma escola também da rede privada de Três de Maio, Rio Grande do $\mathrm{Sul}^{9}$, apresentaram uma prevalência de insatisfação corporal de 85,0\%. Dentre elas, 71,7\% dese- javam reduzir o tamanho corporal e 13,3\% desejavam aumentá-lo. Nos achados do presente estudo, entre as meninas de nível econômico A/B encontrou-se maior prevalência de satisfação com a imagem corporal (42,0\%), e entre aquelas insatisfeitas, $45,4 \%$ gostariam de diminuir a silhueta.

Dessa forma, este estudo apontou notórias diferenças no comportamento dos adolescentes pertencentes aos distintos níveis econômicos quanto à imagem corporal. Meninos e meninas dos níveis econômicos superiores mostraram maior insatisfação com sobrepeso e obesidade do que os provenientes das classes mais baixas e, em todos os níveis econômicos, as meninas são mais descontentes com o excesso de peso e os meninos com a magreza.

A partir do encontrado, esse estudo auxilia na compreensão da relação existente entre a insatisfação corporal e algumas variáveis que são facilmente descritas em estudos populacionais, como o sexo e o nível econômico. Os resultados servem para nortear políticas públicas para a execução de diferentes ações em diferentes grupos específicos, pois como visto a insatisfação corporal não apresenta comportamento homogêneo na população adolescente. Considerar essas especificidades pode ser indispensável para a prevenção do desenvolvimento de problemas psíquicos que estão relacionados à autoimagem e transtornos alimentares. São necessárias mais investigações sobre a imagem corporal e suas nuances entre os indivíduos de diferentes níveis socioeconômicos para tentar compreender melhor os motivos subjacentes de tais diferenças. Estudos de caráter qualitativo também poderiam ajudar a compreender melhor este fenômeno.

\section{Colaboradores}

SC Dumith, AMB Menezes, RM Bielemann, S Petresco, ICM Silva, RS Linhares, TC Amorim, DV Duarte, CLP Araújo e JV Santos participaram igualmente de todas as etapas de elaboração do artigo. 


\section{Agradecimentos}

Este artigo é baseado nos dados do estudo "Coorte de nascimentos de 1993 de Pelotas", realizado pelo Programa de Pós Graduação em Epidemiologia, da Universidade Federal de Pelotas. A visita aos 15 anos foi financiada pela Wellcome Trust Initiative.

\section{Referências}

1. Instituto Brasileiro de Geografia e Estatística (IBGE). Pesquisa Nacional de Saúde do Escolar - 2009. Rio de Janeiro: IBGE; 2009.

2. Conti MA, Frutuoso MFP, Gambardella AMD. Excesso de peso e insatisfação corporal em adolescentes. Rev Nutr 2005; 18(4):491-497.

3. Tabak I, Mazur J, Oblaciñska A, Jodkowska M. Body mass, self-esteem and life satisfaction in adolescents aged 13-15 years. Med Wieku Rozwoj 2007; 11(3Pt1):281-290.

4. Victora CG, Araújo CL, Menezes AMB, Hallal PC, Vieira MF, Neutzling MB, Gonçalves H, Valle NC, Lima RC, Anselmi L, Behague D, Gigante DP, Barros FC. Methodological aspects of the 1993 Pelotas (Brazil) Birth Cohort Study. Rev Saude Publica 2006; 40(1):39-46.

5. Onis M, Onyango AW, Borghi E, Siyam A, Nishida C, Siekmann J. Development of a WHO growth reference for school-aged children and adolescents. Bull World Health Organ 2007; 85(9):660-667.

6. Associação Brasileira de Empresas de Pesquisa (Abep). Critério de Classificação Econômica Brasil 2008. São Paulo: Abep; 2007.

7. Tiggemann M, Wilson-Barrett E. Children's figure ratings: Relationship to self-esteem and negative stereotyping. Int J Eat Disord 1998; 23(1):83-88.

8. Triches R, Giugliani E. Insatisfação corporal em escolares de dois municípios da região Sul do Brasil. Rev Nutr 2007; 20(2):119-128.

9. Corseuil MW, Pelegrini A, Beck C, Petroski EL. Prevalência de insatisfação com a imagem corporal e sua associação com a inadequação nutricional em adolescentes. Rev. Educação Física/UEM 2009; 20(1):25-31.

10. Freitas AR, Novello D, Gastaldon LT, Justino PF. Insatisfação da imagem corporal, práticas alimentares e de emagrecimento em adolescentes do sexo feminino. Rev Bras Nutr Clin 2009; 24(3):166-173.

11. Wang $Y$, Liang $H$, Chen X. Measured body mass index, body weight perception, dissatisfaction and control practices in urban, low-income African American adolescents. BMC Public Health 2009; 9(183).
12. Branco LM, Hilário MOE, Cintra IdP. Percepção e satisfação corporal em adolescentes e a relação com seu estado nutricional. Rev Psiq Clín 2006; 33(6):292296.

13. Haines J, Kleinman KP, Rifas-Shiman SL, Field AE, Austin SB. Examination of Shared Risk and Protective Factors for Overweight and Disordered Eating Among Adolescents. Arch Pediatr Adolesc Med 2010; 164(4):336-343.

14. Jones DC. Body image among adolescent girls and boys: a longitudinal study. Dev Psychol 2004; 40(5):823-835.

15. Instituto Brasileiro de Geografia e Estatística (IBGE). Pesquisa de Orçamentos Familiares 2002-2003: antropometria e análise do estado nutricional de crianças e adolescentes no Brasil. Rio de Janeiro: IBGE; 2006.

16. Carter R, Jaccard J, Silverman W, Pina A. Pubertal timing and its link to behavioral and emotional problems among 'at-risk' African American adolescent girls. J Adolesc 2009; 32(3):467-481.

17. Tanner JM. Foetus into man: Physical growth from conception to maturity. London: Open Books; 1978.

18. Pereira ÉF, Graup S, Lopes AdS, Borgatto AF, Daronco LSE. Percepção da imagem corporal de crianças e adolescentes com diferentes níveis socio-econômicos na cidade de Florianópolis, Santa Catarina, Brasil. Rev Bras Saúde Matern Infant 2009; 9(3):253-262.

Artigo apresentado em 13/05/2011

Aprovado em 12/07/2011

Versão final apresentada em 29/07/2011 